\title{
BIRTH WEIGHT IS ASSOCIATED WITH SIGNIFICANT CLINICAL AND BIOCHEMICAL PARAMETERS OF PCOS
}

Stavroula A. Paschou ${ }^{1}$, Dimitrios Ioannidis ${ }^{2}$, Evangeline Vassilatou ${ }^{3}$, Maria Panagou ${ }^{2}$, Dimitrios Lilis ${ }^{2}$, Ioanna Tzavara $^{2}$, Andromachi Vryonidou ${ }^{1}$

'Department of Endocrinology and Diabetes, Hellenic Red Cross Hospital, Athens, Greece

${ }^{2}$ Department of Endocrinology and Diabetes, "Amalia Fleming” Hospital, Athens, Greece

${ }^{3}$ Endocrine Unit, Second Department of Medicine, “Attikon” University Hospital, Athens, Greece

Introduction: Several studies have demonstrated an association of birth weight with metabolic and reproductive abnormalities in adults. The aim of this study was to investigate the birth weight in women with PCOS and its correlation with other important clinical and biochemical characteristics of the syndrome.

Patients \& Methods: We studied 288 women with PCOS according to the NIH criteria (1990) and 166 women with normal cycles and without clinical hyperandrogenism. Birth Weight, levels of androgens, insulin and fasting glucose were recorded.

Results:

- Patients and controls were matched for age and Body Mass Index (BMI). Women with PCOS had statistically significant increased central obesity and insulin resistance. They also had increased levels of all androgens (Total Testosterone, $\triangle 4 \mathrm{~A}$, and DHEAS) and reduced levels of SHBG.

- Information on birth weight was available for 224/288 women with PCOS and 75/166 controls.

- No differences were found ( $p>0.05)$ in birth weight between women with PCOS $(3.228 \pm 530 \mathrm{gr})$ and normal controls $(3.160 \pm 503 \mathrm{gr})$.

\begin{tabular}{|c|c|c|c|}
\hline & $\begin{array}{l}\text { PCOS Patients } \\
(\mathbf{n}=\mathbf{2 8 8})\end{array}$ & $\begin{array}{l}\text { Controls } \\
(\mathrm{n}=166)\end{array}$ & p value \\
\hline Age(years) & $24.9 \pm 6.1$ & $26 \pm 6.4$ & ns \\
\hline BMI $\left(\mathrm{kg} / \mathrm{m}^{2}\right)$ & $27.9 \pm 7.3$ & $27 \pm 7.6$ & ns \\
\hline Waist Circumference (cm) & $89.9 \pm 18$ & $82.9 \pm 16.8$ & $<0.001$ \\
\hline HOMA-IR & $2.8 \pm 2.1$ & $2.2 \pm 1.5$ & $<0.001$ \\
\hline TTesto (ng/ml) & $0.92 \pm 0.44$ & $0.42 \pm 0.14$ & $<0.001$ \\
\hline$\Delta 4 \mathrm{~A}(\mathrm{ng} / \mathrm{ml})$ & $3.5 \pm 1.3$ & $1.8 \pm 0.5$ & $<0.001$ \\
\hline DHEAS ( $\mu \mathrm{g} / \mathbf{d l})$ & $296.2 \pm 122.8$ & $219.6 \pm 87.8$ & $<0.001$ \\
\hline SHBG (nmol/t) & $31.5 \pm 13.2$ & $52.3 \pm 21.7$ & $<0.001$ \\
\hline Birth Weight (gr) & $\begin{array}{l}3228 \pm 530 \\
\left({ }^{*} \mathrm{n}=224\right)\end{array}$ & $\begin{array}{l}3160 \pm 503 \\
\left({ }^{*} n=75\right)\end{array}$ & ns \\
\hline
\end{tabular}

- In women with PCOS, birth weight was negatively correlated with the levels of DHEAS and positively correlated with waist circumference and BMI.

\begin{tabular}{|c|c|}
\hline Patients & $\begin{array}{l}\text { Birth Weight } \\
\text { (gr) }\end{array}$ \\
\hline BMI $\left(\mathrm{kg} / \mathrm{m}^{2}\right)$ & $\begin{array}{l}\mathrm{p}=0.04 \\
\mathrm{r}=0.136\end{array}$ \\
\hline Waist Circumference $(\mathrm{cm})$ & $\begin{array}{l}\mathrm{p}<0.001 \\
\mathrm{r}=0.312\end{array}$ \\
\hline HOMA-IR & ns \\
\hline TTesto (ng/ml) & ns \\
\hline$\Delta 4 \mathrm{~A}(\mathrm{ng} / \mathrm{ml})$ & ns \\
\hline DHEAS ( $\mu \mathrm{g} / \mathrm{dl})$ & $\begin{array}{l}p=0.04 \\
r=-0.137\end{array}$ \\
\hline SHBG (nmol/lt) & ns \\
\hline
\end{tabular}

- In controls, birth weight was negatively correlated with the levels of SHBG.

\begin{tabular}{|l|l|}
\hline Controls & $\begin{array}{l}\text { Birth Weight } \\
(\mathrm{gr})\end{array}$ \\
\hline BMI $\left(\mathrm{kg} / \mathrm{m}^{2}\right)$ & $\mathrm{ns}$ \\
\hline Weight Circumference $(\mathrm{cm})$ & $\mathrm{ns}$ \\
\hline HOMA-IR & $\mathrm{ns}$ \\
\hline TTesto $(\mathrm{ng} / \mathrm{ml})$ & $\mathrm{ns}$ \\
\hline$\Delta 4 \mathrm{~A}(\mathrm{ng} / \mathrm{ml})$ & $\mathrm{ns}$ \\
\hline DHEAS $(\mu \mathrm{g} / \mathrm{dl})$ & $\mathrm{ns}$ \\
\hline SHBG $(\mathrm{nmmol} / \mathrm{lt})$ & $\begin{array}{l}\mathrm{p}=0.026 \\
\mathrm{r}=-0.263\end{array}$ \\
\hline
\end{tabular}

$\checkmark$ Then, we divided women from both two groups in 6 categories according to birth weight. We observed no statistically significant differences in the distribution percentages among those with PCOS and normal controls.

\begin{tabular}{|l|l|l|l|}
\hline Birth Weight & $\begin{array}{l}\text { Patients } \\
\left({ }^{*} \mathrm{n}=224 / 288\right)\end{array}$ & $\begin{array}{l}\text { Controls } \\
\left({ }^{\star} \mathrm{n}=75 / 166\right)\end{array}$ & p value \\
\hline$<2.500 \mathrm{gr}$ & $8 \%$ & $12 \%$ & $\mathrm{~ns}$ \\
\hline $2.501-3.000 \mathrm{gr}$ & $27.8 \%$ & $22.7 \%$ & $\mathrm{~ns}$ \\
\hline $3.001-3.500 \mathrm{gr}$ & $38.4 \%$ & $40 \%$ & $\mathrm{~ns}$ \\
\hline $3.501-4.000 \mathrm{gr}$ & $21.4 \%$ & $25.3 \%$ & $\mathrm{~ns}$ \\
\hline $4.001-4.500 \mathrm{gr}$ & $4.5 \%$ & $0 \%$ & $\mathrm{~ns}$ \\
\hline$>4.500 \mathrm{gr}$ & $0.9 \%$ & $0 \%$ & $\mathrm{~ns}$ \\
\hline
\end{tabular}

Conclusions: We did not find any differences in birth weight between women with PCOS and normal controls. However, birth weight was associated with significant clinical and biochemical parameters of PCOS, including obesity and hyperandrogenism. 